Check for updates

Cite this: RSC Adv., 2019, 9, 37705

Received 8th October 2019

Accepted 6th November 2019

DOI: 10.1039/c9ra08166f

rsc.li/rsc-advances

\section{Resonance energy transfer-assisted random lasing in light-harvesting bio-antenna enhanced with a plasmonic local field $\dagger$}

\begin{abstract}
Partha Kumbhakar, Subrata Biswas and Pathik Kumbhakar iD *
Thanks to the advent of the random laser, new light applications have opened up, ranging from biophotonic to security devices. Here, by using the well-known but unexplored light-harvesting bio-pigment of butterfly pea (Clitoria ternatea, CT) flower extract, generation of continuous-wave (CW) random lasing at $\sim 660 \mathrm{~nm}$ has been demonstrated. Furthermore, a wavelength tunability of $\sim 30 \mathrm{~nm}$ in the lasing emission was obtained by utilizing the resonance energy transfer (RET) mechanism in a gain medium with a binary mixture of CT extract and a commercially available methylene blue (MB) dye as the gain medium. In the CT extract-dye mixture, the bio-pigments are acting as donors and the MB dye molecules are acting as acceptors. Amplification in intensity of the lasing emission of this binary system has further been achieved in the presence of optimized concentrations of metal $(\mathrm{Ag})$-semiconductor $(\mathrm{ZnO})$ scattering nanoparticles. Interestingly, the lasing threshold has been reduced from 128 to $25 \mathrm{~W} \mathrm{~cm}^{-2}$, with a narrowed emission peak just after loading of the Ag nanoplasmon in the $\mathrm{ZnO}$-doped binary gain medium. Thanks to the strong localized electric field in the metal nanoplasmon, and the multiple scattering effects of $\mathrm{ZnO}$, the lasing threshold was reduced by approximately four times compared to that of the gain medium without the use of scatterers. Thus, we believe that our findings on wavelengthtunable, non-toxic, biocompatible random lasing will open up new applications, including the design of low-cost biophotonic devices.
\end{abstract}

\section{Introduction}

The laser, almost 60 years after its invention, has become a key light source in modern imaging systems. However, a conventional laser has a high degree of spatial coherence, which produces unwanted coherent imaging artefacts, such as the formation of speckles. Therefore, researchers are now putting enormous effort into devising an unconventional laser system, called a random laser (RL), which is free from such drawbacks. ${ }^{1-3}$ Demonstrations of random lasing have been reported with both pulsed and continuous-wave (CW) pumping. ${ }^{4-12}$

Generally, long-wavelength or near-infrared (NIR) (>650 nm) fluorescent dyes have high penetration properties in tissue. ${ }^{13-15}$ Therefore, they can be fruitfully used for biomedical and biophotonic applications, such as in surgical therapy treatments, cancer diagnostics, etc. ${ }^{13-16}$ However, these dyes have some drawbacks, including poor photostability, low absorption at specific pump wavelength, non-biodegradability, toxicity, and also expense. ${ }^{1,13-16}$ Therefore, they are not appropriate in

Nanoscience Laboratory, Department of Physics, National Institute of Technology, Durgapur, 713209, India. E-mail: pathik.kumbhakar@phy.nitdgp.ac.in; nitdgpkumbhakar@yahoo.com

$\dagger$ Electronic supplementary information (ESI) available. See DOI: 10.1039/c9ra08166f biomedical and biophotonics applications. Recently, environmentally friendly, non-toxic, optically active bioorganic dyes extracted from natural resources, such as carotene, porphyrins, chlorophylls, etc., have become the obvious choice as the gain medium in RL generation. Use of these dyes has many advantages; $;^{\mathbf{8 1 0 , 1 7 , 1 8}}$ for example, they (1) are biodegradable and biocompatible, (2) have efficient light-harvesting capabilities in the long-wavelength region, and (3) have higher molar extinction coefficients to enable efficient light harvesting. Additionally, these bio-pigments are available in large quantity, and can be safely extracted from fruits, flowers and leaves. ${ }^{8-10,17-20}$ Here, we have selected a biocompatible natural organic dye, namely extract of butterfly pea (Clitoria ternatea, CT), a medicinal plant, which is used extensively as a natural colouring agent around the world. ${ }^{17}$ This flower extract has already been used as a substitute for commercial toxic blue dye in different medical and food packaging sectors. ${ }^{18,19}$ The blue colour of the extract in neutral aqueous solution indicates the presence of different flavonoid and anthocyanin content, as reported by others. ${ }^{18,19}$ Such optically active biodegradable organic dyes provide a unique possibility for RL generation in combination with metal/semiconductor nanoparticles (NPs) as the scattering particles..$^{4-6,21-23}$ Traditionally, it has been reported that, RL generation is dependent on multiple scattering of light in a random gain medium. The presence of the strong electric field 
of metal NPs may enhance these scattering properties, and may also modify the transition rates of nearby dye molecules. ${ }^{4-6}$ Random lasing has been obtained in several materials, such as metal/semiconductor NPs, human tissue, liquid crystals, fluorescent polymers, commercial dyes, etc..$^{4-7,21-23}$

At present, there is a huge demand for multi-coloured random laser sources in the medical and optoelectronics sectors. The resonance energy transfer (RET) process is another strategy to achieve tunable emission, with improved emission intensity from a gain media. ${ }^{24-27}$ The minimum requirement for the occurrence of energy transfer in the RET process is the availability of good overlap between the emission band of the donor and the absorption band of the acceptor. ${ }^{4}$ One example, is a mixture system with more than one dye, in which one (donor) dye efficiently absorbs the pump radiation, and then the excitation energy is non-radiatively transferred to the second (acceptor) dye. ${ }^{\mathbf{6} 24-27}$

Here, we demonstrate RET-assisted CW RL generation using a bioorganic pigment as the gain medium, which is biodegradable and cheap. Also, we have improved the lasing efficiency and achieved tunable emissions by employing a RET process in which CT dye molecules transfer energy to methylene blue (MB) dye. The mechanism of the energy transfer has also been studied by analyzing the optical properties of the mixtures of donor and acceptor after encapsulation in a polymer film. A significant reduction in lasing threshold, from 128 to $25 \mathrm{~W}$ $\mathrm{cm}^{-2}$, has been demonstrated, with systematic increase in the number and concentration of scatterers ( $\mathrm{ZnO}$ and $\mathrm{Ag}$ NPs). Thorough analysis of the RL emission allowed us to infer the role of the energy transfer process and the presence of semiconductor and plasmonic NPs in enhancing the lasing emission. The unprecedented demonstration of RET-assisted tunable random lasing in this work indicates that natural pigments can be effectively utilized to develop cost-effective random lasers using easy processing steps.

\section{Results and discussion}

The UV-visible absorption spectrum of the bio-pigment was measured and is shown in Fig. 1a. The presence of Q-bands at $\sim 533 \mathrm{~nm}, \sim 573 \mathrm{~nm}$, and $\sim 618 \mathrm{~nm}$ are clearly seen in Fig. 1a. We also studied the photoluminescence (PL) emission spectrum of the extracted pigment (Fig. 1a), which was found to be consistent with the visible PL emission peak at $\sim 660 \mathrm{~nm}$ corresponding to the $S_{1} \rightarrow S_{0}$ transition. Digital photographs of the butterfly pea and the extraction of pigments in water are also shown in Fig. 1a (right inset). To determine the presence of different functional groups in the organic pigments, Fouriertransform infrared spectroscopy (FTIR) data were collected and are shown in Fig. 1b. From Fig. 1b it can be seen that several functional groups, such as $-\mathrm{CH},-\mathrm{C}=\mathrm{O},-\mathrm{OH}$, are present in the bio-pigment. The presence of hydroxyl groups (Fig. 1b) helps the anthocyanins to absorb light in the visible region. ${ }^{18}$ The existence of a strong absorption band at $\sim 632 \mathrm{~nm}$ and a good PL emission peak at $\sim 660 \mathrm{~nm}$ facilitated the use of this biopigment as a gain medium in the $\mathrm{RL}$ experiment, as shown later. The PL emission of the pigment in the solution phase was measured at different input pump intensities (IP) of a $\mathrm{He}-\mathrm{Ne}$ laser with $\lambda_{\text {pump }}=632.8 \mathrm{~nm}$, as shown schematically in Fig. 1c. The results obtained are shown in Fig. 1d and e. Although amplified spontaneous emission (ASE) was observed, narrowing of the spectral profile, as well as a distinct threshold, was not observed, even after increasing the pump intensity.

Therefore, to overcome such drawbacks, i.e. low laser emission at NIR region as well as tunability in lasing emission, the RET process has been employed as an important strategy. It was found that there is good overlap between the emission band of CT (solid red line) and the absorption band of MB (solid black line) (Fig. 1f), confirming the possibility of efficient energy transfer (ET) from the donor (CT) to the acceptor (MB) dye. The calculated value of the overlap integral is $J(\lambda)=2.94 \times 10^{15}$ $\mathrm{nm}^{4} \mathrm{M}^{-1} \mathrm{~cm}^{-1}$ using eqn (1),

$$
J(\lambda)=\frac{\int_{0}^{\infty} F_{\mathrm{D}}(\lambda) \varepsilon_{\mathrm{A}}(\lambda) \lambda^{4} \mathrm{~d} \lambda}{\int_{0}^{\infty} F_{\mathrm{D}}(\lambda) \mathrm{d} \lambda}
$$

where $F_{\mathrm{D}}(\lambda)$ is the total normalized PL intensity (area under the curve) of pigments in the wavelength range $\lambda+\Delta \lambda$ and $\varepsilon(\lambda)$ is the molar extinction coefficient of the MB dye at $\lambda$.

The origin of the emission tunability and details of the tuning mechanism for the lasing emission using the RET process are illustrated schematically in Fig. 1g. The input energy of the pump light $(632.8 \mathrm{~nm})$ is mostly absorbed by CT molecules. The excited CT molecules partially transfer their energy to the $\mathrm{MB}$ molecules, leading to the dual-colour emission at $\sim 660 \mathrm{~nm}$ (near red) and $\sim 692 \mathrm{~nm}$ (deep red), respectively. It was found that the output emission intensity from the donor increased rapidly once the pump intensity exceeded the lasing threshold energy, while some residual acceptor emission was also found to increase slowly. As the acceptor/donor (A/D) ratio increased further, the lasing threshold of the acceptor showed a stable output from the donor/acceptor mixture system. Furthermore, after addition of semiconductor and metal NPs as scatterers in the random system, multiple scattering significantly disturbed the random system and decreased the lasing threshold, by dynamically changing the strong local electromagnetic field. ${ }^{4,5}$ Thus, the RET process greatly influences the multi-colour RL under single excitation conditions. In addition, we have measured stability of the MB dye with laser exposure time (Fig. S1 $\dagger$ ) and it was clearly seen that there is almost no change in emission intensity after laser irradiation for a duration up to $15 \mathrm{~min}$ (for details see ESI $\dagger$ ), and only a $10 \%$ reduction in intensity after $45 \mathrm{~min}$.

Fig. 2a represents the PL emission spectra of the RET-based binary system with different volume ratios of the acceptor and donor. Two clear emission bands (red and cyan arrow) for the $\mathrm{CT}$ and $\mathrm{MB}$ dyes, centred at $\sim 660 \mathrm{~nm}$ and $\sim 692 \mathrm{~nm}$, are observed under $632.8 \mathrm{~nm}$ excitation. The A/D ratio was changed in order to control the RET efficiency (Fig. 2b). A coefficient known as the energy transfer coefficient $\left(\eta_{\mathrm{ET}}\right)^{27}$ was calculated using eqn (2), to describe the ET processes physically in the semiconductor-metal-based binary random lasing system. The expression for $\eta_{\mathrm{ET}}$ is given as, 

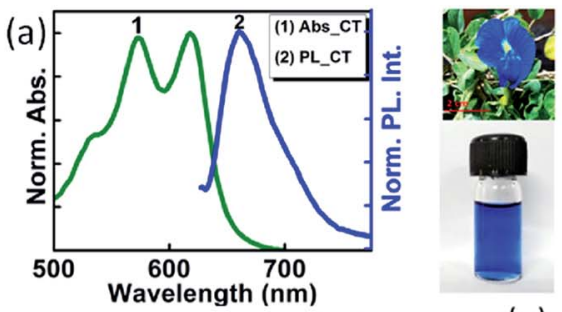

(b) 웅

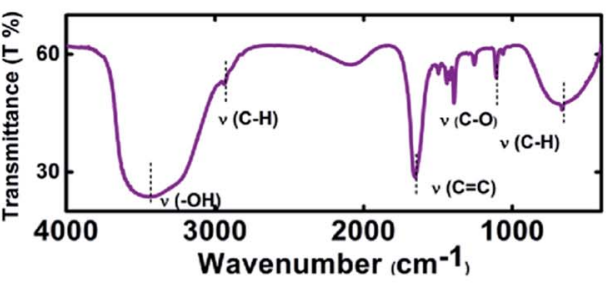

(c)

(d)
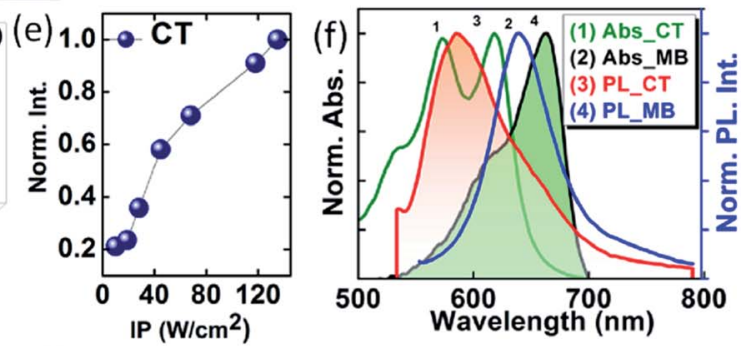

(g)

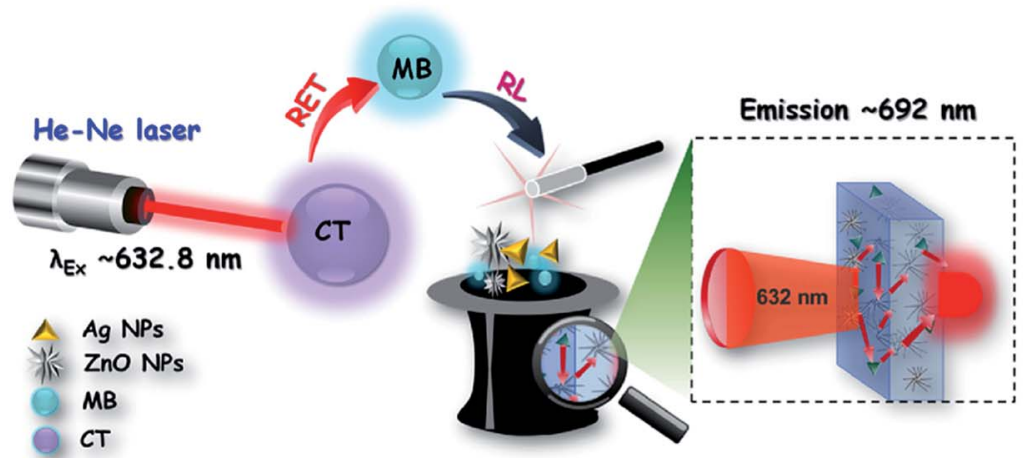

Fig. 1 (a) UV-visible absorption and PL emission spectra of natural CT dye extract. Right inset (upper panel) shows digital photographs of the flower and its extraction in water (lower panel). (b) FTIR spectrum of CT. (c) A schematic illustration of CW excitation and PL emission of the biopigment in solution phase. (d) Emission spectra of the CT dye extract without scatterers at different IP intensities. (e) Emission intensity from CT excited by $632.8 \mathrm{~nm}$ under different IP without scatterers. (f) The normalized absorption spectra (1 and 2) and the PL emission spectra (3 and 4 ) of $\mathrm{CT}$ and $\mathrm{MB}$ dye in water. (g) A schematic illustration of the RET process in the metal-semiconductor-based dual-colour random laser under CW pumping.

$$
\eta_{\mathrm{ET}}=I_{\mathrm{A}} /\left(I_{\mathrm{A}}+I_{\mathrm{D}}\right)
$$

where $I_{\mathrm{A}}$ is the integrated emission intensity of $\mathrm{MB}$ dye at $\sim 692 \mathrm{~nm}$ and $I_{\mathrm{D}}$ is the integrated emission intensity of CT at $\sim 660 \mathrm{~nm}$. It was found that, as we increased the A/D ratio from 0 to 7.33 , the intensity of the emission band corresponding to CT quenches, while the intensity of the emission corresponding to the MB dye increases gradually under excitation at $632 \mathrm{~nm}$ (Fig. S2 $\uparrow$ ). The evolution of $\eta_{\mathrm{ET}}$ with $\mathrm{A} / \mathrm{D}$ ratio is demonstrated in Fig. 2 b. As the A/D ratio increases from 0.85 to 19.0 , the RET coefficient is also found to increase and reaches saturation at A/ $\mathrm{D}=7.33$ (Fig. 2b). ${ }^{25}$

The PL emission spectra of the bio-pigment in the presence of scatterers with appropriate concentrations are shown in Fig. 3. The microstructural properties of rod-like $\mathrm{ZnO}$ and triangular Ag NPs are shown in the typical SEM and TEM images in Fig. 3a and b. The absorption spectra of ZnO and Ag NPs can also be seen to have a spectral overlap with the emission spectra of the bio-pigment, as shown in Fig. 3c. The presence of a broad absorption peak at $\sim 600 \mathrm{~nm}$ is due to the in-plane dipole resonance in the triangular $\mathrm{Ag} \mathrm{NPs}^{8}{ }^{8}$ The large scattering property of ZnO NPs, which is due to the contrast in refractive index in the gain medium, provides multiple scattering leading to the observation of ASE. ${ }^{27-29}$ The results are shown in Fig. 3d and indicate that the amplification is not sufficient to achieve proper lasing emission at low pumping energy using only $\mathrm{ZnO}$ NPs. Therefore, to achieve further increase in the output
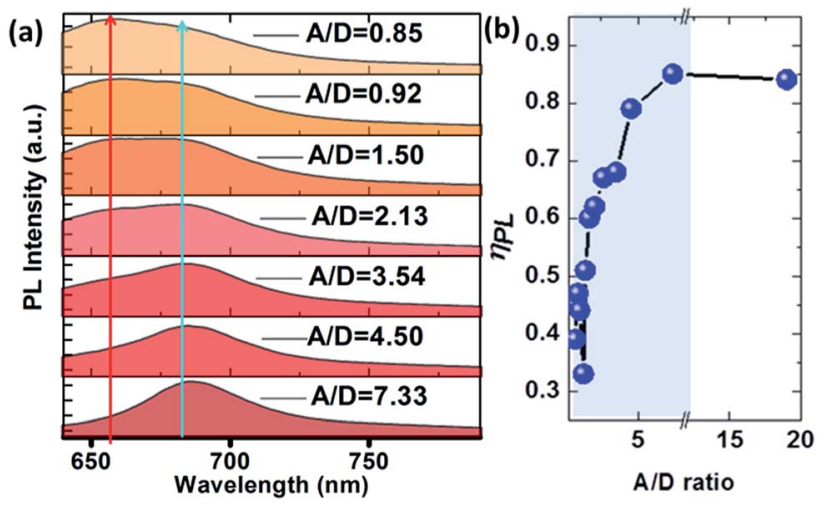

Fig. 2 (a) Normalized PL emission spectra of $C T$ and $M B$ dye with different $A / D$ ratios. (b) Effective RET efficiency as a function of $A / D$ ratio in the binary system. 

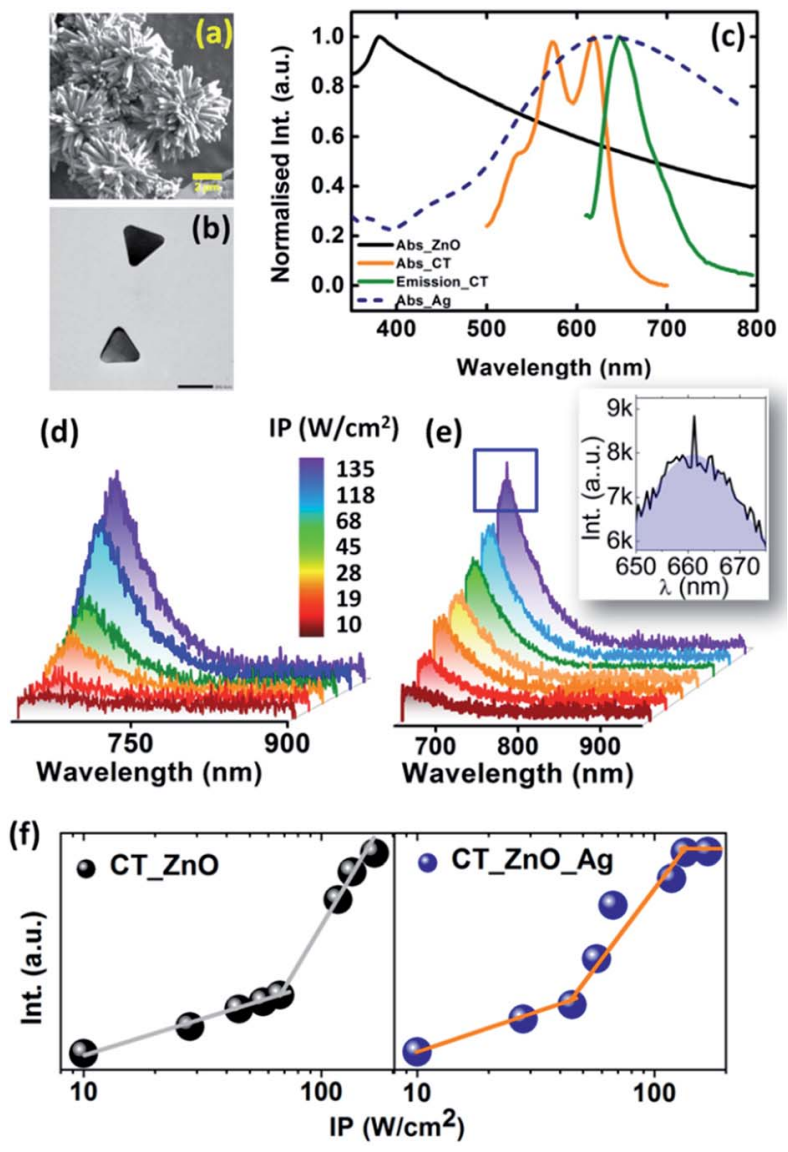

Fig. 3 (a) SEM image of ZnO NPs. (b) TEM image of triangular Ag NPs. (c) Normalized absorption spectra of $\mathrm{ZnO}$ and Ag NPs with absorptionemission spectra of the CT dye. Emission spectra of CT dye extract in the presence of (d) ZnO only and (e) ZnO-Ag NP scatterers at different input intensities. The inset shows an enlarged section of the emission pattern, where we can easily observe generation of the RL mode. (f) Emission intensity from CT-ZnO (left) and CT-ZnO-Ag (right) solution excited by $632.8 \mathrm{~nm}$ under different input intensities.

intensity of the solution, metal NPs were used as an additional scatterer to provide strong, multiple scattering through enhancement of the electromagnetic field (Fig. 3e). The changes in emission intensity with a fixed concentration ratio of $\mathrm{ZnO}$ and triangular-shaped Ag NPs are shown in Fig. 3e and it can be seen that effective light amplification takes place and some lasing peaks have appeared on top of the emission band (inset in Fig. 3e). The dependence of the integrated normalized emission intensity of the pigments on input pump intensity, in the presence of scatterers, is shown in Fig. 3f. From Fig. 3f, a lasing threshold $\left({ }^{\mathrm{CW}} \mathrm{I}_{\mathrm{th}}\right.$ ) of $128 \mathrm{~W} \mathrm{~cm}^{-2}$ was obtained after addition of an optimized concentration of $\mathrm{ZnO}\left(n_{\mathrm{ZnO}}=7 \times 10^{12}\right.$ $\left.\mathrm{ml}^{-1}\right)$ and $\mathrm{Ag}$ NPs $\left(C_{\mathrm{Ag}}=12 \times 10^{15} \mathrm{ml}^{-1}\right)$ in the random system.

It can be seen from the plot (Fig. 3f, left) that within the pump energy used there is only one kink when ZnO NPs are used as the scatterer in the CT dye. However, two kinks are clearly visible (Fig. 3f, right) in the presence of the binary scatterer, i.e. $\mathrm{ZnO}$ and $\mathrm{Ag}$ NPs in the CT dye, ${ }^{3}$ indicating the transition from spontaneous emission (SE) to amplified spontaneous emission (ASE) and to lasing. Therefore, from the results shown in Fig. $3 \mathrm{f}$ (right), it can be concluded that, even with the low pump energy used, lasing is observed in the CT dye by using the binary scatterer, since the metal NPs have caused further enhancement of the electromagnetic field of the pump light by surface plasmon resonance processes.

To demonstrate RL generation in the near-IR region, with significant improvement in the lasing emission of the gain medium, the dye mixture was encapsulated in a homogeneously dispersed aqueous solution of a polymer matrix, as shown in Fig. 4a (details of the procedure are in given in the Experimental section). Fig. 4b shows the emission spectra of samples with optimized scatterer concentrations at two input pump intensities of $10 \mathrm{~W} \mathrm{~cm}^{-2}$ and $135 \mathrm{~W} \mathrm{~cm}^{-2}$. Depending on the different $\mathrm{A} / \mathrm{D}$ ratios with energy transfer efficiency, different scenarios have been observed. At low $\mathrm{A} / \mathrm{D}$ concentration ratio $(\mathrm{A} / \mathrm{D}=0.85)$, the emission peak intensity of the CT dye at $\sim 660 \mathrm{~nm}$ is found to be stronger than that of the emission peak intensity of the MB dye at $\sim 692 \mathrm{~nm}$. As the value of IP increases, from 10 to $135 \mathrm{~W} \mathrm{~cm}^{-2}$, a large number of lasing peaks appear on top of the emission band at $\sim 660 \mathrm{~nm}$, which is clear from Fig. $4 \mathrm{~b}$, and those peaks fluctuate randomly. ${ }^{6,30-32}$ On the other hand, a relatively low, intense, broad emission band for the MB dye is observed at $\sim 692 \mathrm{~nm}$. Upon further increase in the $\mathrm{A} / \mathrm{D}$ ratio (from 1.5 to 7.33 ), more energy is non-radiatively transferred to the acceptor, which prevents lasing of the donor and helps spectral narrowing of the acceptor (Fig. 4b). ${ }^{33}$ Therefore, it can be concluded that the emission intensity ratios are greatly influenced by the variation in $\mathrm{A} / \mathrm{D}$ ratio in such random binary systems. Additionally, for a fixed A/D ratio, the appearance of a number of sharp peaks increased with increasing IP value, indicating the occurrence of $\mathrm{RL}$ at high values of input energy. It can be seen from Fig. $4 \mathrm{~b}$ that there are some random spikes appearing that cover the wavelength range beyond $700 \mathrm{~nm}$, and at around $750 \mathrm{~nm}$ when the pump intensity is at its highest. From static PL emission measurements of the MB dye only, it was found that some shoulder peaks appear at $\sim 750 \mathrm{~nm}$; so, the peaks at $\sim 750 \mathrm{~nm}$ in Fig. $4 \mathrm{~b}$ might have appeared due to emission from the MB dye. However, further studies are required to find the exact origin of peaks other than those appearing at $\sim 660 \mathrm{~nm}$ and $\sim 692 \mathrm{~nm}$. Continuing our study of the switching of emission with $\mathrm{A} / \mathrm{D}$ ratio, the change in CIE colour chromaticity with increasing A/D has also been calculated, as shown in Fig. 4c. In the CIE 1931 colour diagram, the circles describe the calculated colour chromaticity of the binary mixture at different $\mathrm{A} / \mathrm{D}$ ratios (Fig. 4c). It was observed that, with increasing $\mathrm{A} / \mathrm{D}$ ratio, the chromaticity values of the multi-colour $R L$ varied from $(0.69,0.29)$ to $(0.54,0.21)$ under the same pumping energy of $135 \mathrm{~W} \mathrm{~cm}^{-2}$. The results shown in Fig. $4 d$ were obtained by multi-peak fitting of the corresponding emission spectra. In Fig. 4d, the transition from SE to ASE and then to random lasing emission in the binary system can be seen. However, the position of the random lasing emission is solely dependent on the $\mathrm{A} / \mathrm{D}$ ratio. At low $\mathrm{A} / \mathrm{D}$ ratio $(0.85)$, the binary system shows random lasing emission at $\sim 660 \mathrm{~nm}$, corresponding to the CT dye with two clear kinks, whereas, at this ratio, the emission from the MB dye at $\sim 692 \mathrm{~nm}$ is of a spontaneous type (Fig. 4d). On the other hand, at high $\mathrm{A} / \mathrm{D}$ ratio (4.50), the binary system exhibits random lasing emission at $\sim 692 \mathrm{~nm}$ due to energy 



Fig. 4 (a) Representation of the preparation process for the polymer films. The resulting composite film is then pumped using a CW He-Ne laser $\left(\lambda_{\text {pump }}=632.8 \mathrm{~nm}\right)$ and the emission from the film is collected and sent to a spectrometer with a detector for analysis. (b) Normalized emission spectra from random systems with different A/D ratio of (i) 0.85 , (ii) 1.50, (iii) 4.50, (iv) 7.33, are excited by $632.8 \mathrm{~nm}$ under low and high IP energy. The concentration of the $\mathrm{ZnO}$ and Ag NP suspensions are $7 \times 10^{12} \mathrm{ml}^{-1}$ and $12 \times 10^{15} \mathrm{ml}^{-1}$, respectively. (c) Switching of colour chromaticity of the emission spectra with different A/D ratio, extracted from the spectra in Fig. 2a. (d) Variation of lasing intensity with different IP at the two peaks of CT ( $660 \mathrm{~nm})$ and MB ( 692 nm) at A/D ratios of 0.85 (upper panel) and 4.50 (lower panel), and observation of their threshold behaviour. (e) The variation in emission intensity of the observed narrow spikes (at $\sim 660 \mathrm{~nm}$ and $692 \mathrm{~nm}$ ) with the number of measurements carried out in a sample with optimized A/D ratios and for a fixed excitation energy of $135 \mathrm{~W} \mathrm{~cm}^{-2}$.

transfer from the CT dye to the MB dye. To study the changes in RL emission intensity, if any, with respect to the number of measurements, the lasing emission spectra in the gain media with optimized A/D ratios of 0.85 and 4.50 were collected up to 95 times at a fixed pump laser excitation intensity of $135 \mathrm{~W} \mathrm{~cm} \mathrm{~cm}^{-2}$. The corresponding results for the two narrow spikes obtained at $\sim 660 \mathrm{~nm}$ and $\sim 692 \mathrm{~nm}$ with an $\mathrm{A} / \mathrm{D}$ ratio of 0.85 and 4.50, respectively, are shown in Fig. 4e. It can be seen that the fluctuations in peak intensity from their average values at $\sim 660 \mathrm{~nm}$ and $692 \mathrm{~nm}$ are very small. However, it was found that the RL peak at $660 \mathrm{~nm}$ was the most intense for an $\mathrm{A} / \mathrm{D}$ ratio of 0.85 , whereas the peak appearing at $692 \mathrm{~nm}$ was the most intense for an $\mathrm{A} / \mathrm{D}$ ratio of 4.50. So, this indicates that the peak intensity at $\sim 692 \mathrm{~nm}$ can be enhanced with increasing $\mathrm{A} / \mathrm{D}$ ratio. Thus, switching of the lasing emission can be achieved by varying the mixing ratio of the donor and acceptor dyes.

In order to study the dependency on the direction of the lasing emission from the polymer film, lasing was measured at different emission angles. Fig. 5a shows a schematic of the position of the detector for collecting the emission spectra at different angles $\left(\theta=0\right.$ to $\left.180^{\circ}\right)$ with respect to the incident $\mathrm{CW}$ excitation. Fig. $5 \mathrm{~b}$ shows the polar graph of the integrated emission intensity versus the detection angles $(\theta)$ of the lasing emission and it can be seen that the emission intensity depends strongly on the angle. It was observed that the lasing emission intensity is highest at $\theta \cong 60^{\circ}$. Therefore, subsequently, the RL emission data have only been collected at an angle of $60^{\circ}$.
In an optical gain medium, disordered structure can offer random lasing via multiple scattering in the medium by changing the scattering length, and this conclusion has been supported by measurement of the mean free path of the binary system. The minimum mean free path $\left(l_{\min }\right)$ of the photons in the polymer film was calculated using eqn (3), ${ }^{30}$

$$
l_{\min }=2 n^{2} d / \sqrt{n^{2}-1},
$$

where, $n$ and $d$ are the refractive index and thickness, respectively, of the CT-MB-ZnO composite polymer film, and the value of $l_{\min }$ was found to be $\sim 0.28 \mathrm{~mm}$. As reported previously,



Fig. 5 (a) Schematic representation showing the detection of lasing emission at different angles. (b) Polar graph of integrated emission intensity at different detection angles. 
random lasing is also observed in a weakly scattering regime $\left(k l_{\min } \gg 1\right) \cdot .^{34,35}$ Therefore, our system has acted as an extremely weak scattering medium $\left(l_{\min } \sim 0.28 \mathrm{~mm}\right)$, which does not help to achieve band narrowing or distinguish threshold behaviour as expected. Therefore, we introduced a plasmonic enhancer (i.e. triangular Ag NPs) into the random system, which can greatly enhance the local electrical field..$^{5,6,35}$ In order to investigate the significance of $\mathrm{Ag}$ NPs on the random system, different concentrations of $\mathrm{ZnO}$ and Ag NPs were carefully mixed in the polymer matrix with different donor-acceptor ratios. Fig. $6 a$ and $b$ shows the RL emission spectra of the polymer films obtained under different input intensity in the absence and presence of Ag NPs, but for the same number density of ZnO NPs. To determine the dependence of the scatterer concentration on ${ }^{{ }^{C W}} \mathrm{I}_{\mathrm{th}}$, the number density of the $\mathrm{ZnO}$ NPs $\left(n_{\text {Zno }}\right)$ was varied from $2 \times 10^{12}$ to $20 \times 10^{12} \mathrm{ml}^{-1}$, as shown in Fig. $6 \mathrm{a}$ and S3. $\dagger$ It was observed that, with the increase in the number density of ZnO NPs from $2 \times 10^{12}$ to $20 \times 10^{12} \mathrm{ml}^{-1}$, ${ }^{\mathrm{CW}} \mathrm{I}_{\text {th }}$ was reduced significantly, from $107 \mathrm{~W} \mathrm{~cm}^{-2}$ to $43 \mathrm{~W} \mathrm{~cm}^{-2}$, as shown in Table 1 and Fig. S3. $\dagger$ The dependence of the input pump energy on the integrated emission intensity in the CTMB-ZnO system (with $n_{\mathrm{ZnO}}=20 \times 10^{12} \mathrm{ml}^{-1}$ ), as depicted in the inset in Fig. 6a, shows a typical ASE nature, with a threshold value of $43 \mathrm{~W} \mathrm{~cm}^{-2}$. In the case of $\mathrm{CW}$ operation, ${ }^{\mathrm{CW}} \mathrm{I}_{\mathrm{th}}$ was not
Table 1 Experimentally obtained values of lasing threshold of the binary mixture

\begin{tabular}{|c|c|c|c|c|c|c|c|}
\hline \multirow[b]{2}{*}{ Concentrations } & \multicolumn{4}{|c|}{$\begin{array}{l}\text { CT-MB and } \mathrm{ZnO} \\
\left(\times 10^{12} \mathrm{ml}^{-1}\right)\end{array}$} & \multicolumn{3}{|c|}{$\begin{array}{l}\mathrm{CT}-\mathrm{MB}-\mathrm{ZnO} \\
\left(n_{\mathrm{ZnO}}=20 \times 10^{12}\right. \\
\left.\mathrm{ml}^{-1}\right) \text { and } \mathrm{Ag} \mathrm{NP} \\
\left(\times 10^{15} \mathrm{ml}^{-1}\right)\end{array}$} \\
\hline & 2 & 7 & 13 & 20 & 12 & 24 & 36 \\
\hline${ }^{\mathrm{CW}} \mathrm{I}_{\mathrm{th}}\left(\mathrm{W} \mathrm{cm}^{-2}\right)$ & 107 & 83 & 60 & 43 & 35 & 33 & 25 \\
\hline
\end{tabular}

as distinct as it is often observed to be during pulsed mode operation, since under $\mathrm{CW}$ conditions spontaneous emission may significantly influence the threshold. ${ }^{36}$

However, with the addition of $\mathrm{Ag}$ NPs, ${ }^{\mathrm{CW}} \mathrm{I}_{\text {th }}$ is reduced significantly, as shown in Fig. 6b. It can be clearly seen that the emission spectra of the polymer film with Ag NPs has multiple peaks, as shown in Fig. $6 \mathrm{~b}$, with the central lasing peak at $\sim 692.4 \mathrm{~nm}$ narrowed down compared with that without Ag NPs (Fig. 6a) for the same pumping energy. The lasing threshold value of the random system has decreased by up to four orders of magnitude due to the presence of Ag NPs (Fig. S3 $\uparrow$ and Table 1). This result indicates the occurrence of random lasing in the presence of Ag NPs, and the lasing modes are generated due to
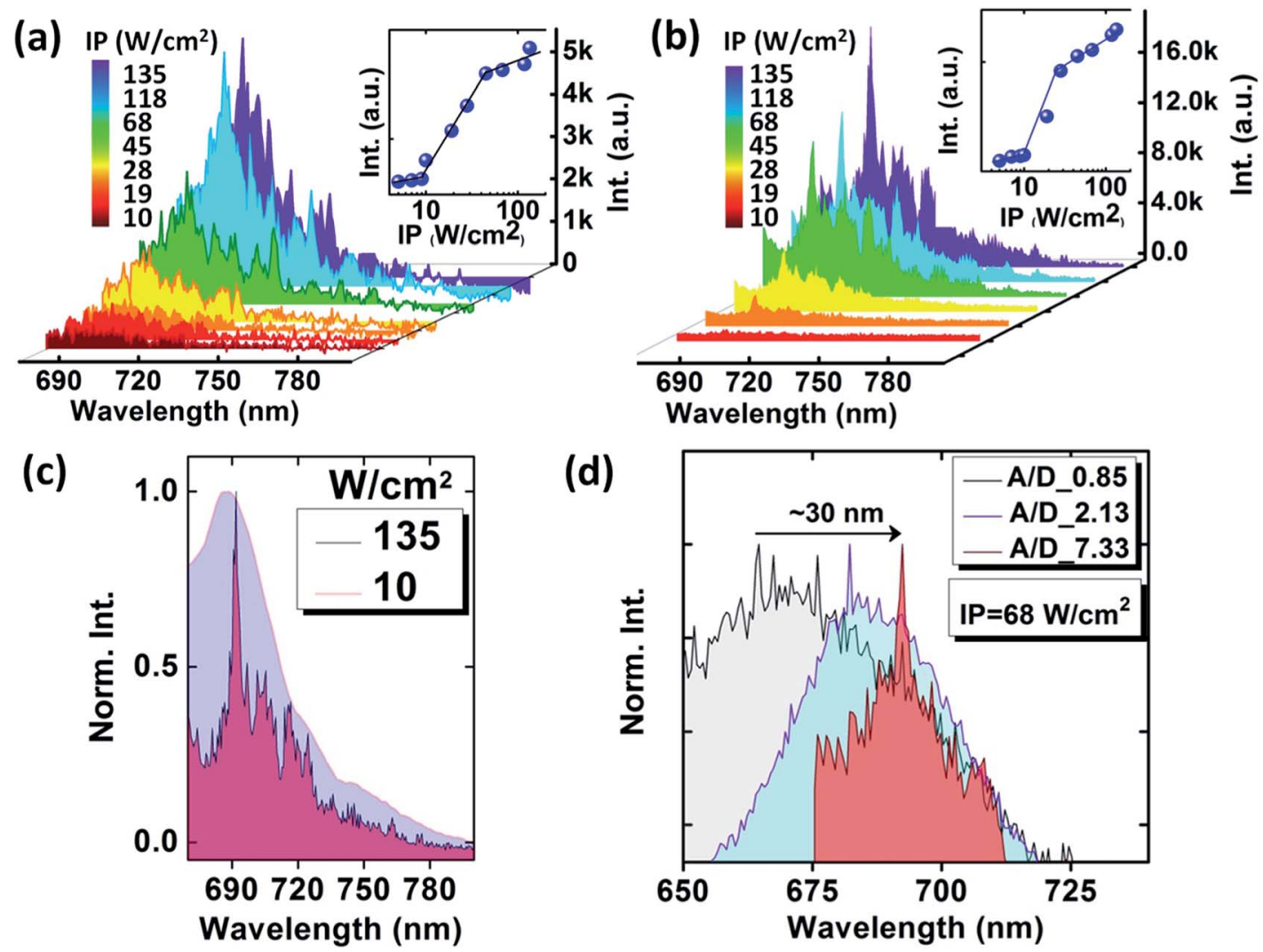

Fig. 6 The emission spectra of CT-MB medium in the presence of the $\mathrm{ZnO}$ scatterer $\left(n_{\mathrm{ZnO}}=7 \times 10^{12}\right)$ at different IP energies (a) without Ag NPs and (b) with Ag NPs $\left(n_{\mathrm{ZnO}}=20 \times 10^{12}\right.$ and $\left.C_{\mathrm{Ag}}=36 \times 10^{15} \mathrm{ml}^{-1}\right)$, respectively. The inset shows the threshold behaviour of the random system for $\mathrm{RL}$ generation in the MB dye. (c) Normalized emission spectra of polymer films containing a mixture of CT and MB dye with ZnO $\left(n_{\mathrm{ZnO}}=20 \times\right.$ $10^{12} \mathrm{ml}^{-1}$ ) and Ag NPs $\left(C_{\mathrm{Ag}}=36 \times 10^{15} \mathrm{ml}^{-1}\right.$ ) under low (red line) and high (black line) IP energy. (d) RL generation in polymer film with three different $A / D$ ratios and in the presence of $\mathrm{ZnO}\left(n_{\mathrm{ZnO}}=20 \times 10^{12} \mathrm{ml}^{-1}\right)$ and Ag NPs $\left(C_{\mathrm{Ag}}=12 \times 10^{15} \mathrm{ml}^{-1}\right)$ under an IP energy of $68 \mathrm{~W} \mathrm{~cm}{ }^{-2}$. 
multiple scattering of trapped photons and enhancement of the local field inside the gain medium. ${ }^{5,6,24-26}$ The narrowed, sharp emission occurring at $\sim 692.4 \mathrm{~nm}$ (Fig. $6 \mathrm{c}$ ) in CT-MB-ZnO-Ag was obtained after the input energy exceeded the threshold value. When the input pump intensity exceeds the threshold, the bandwidth of the central peak becomes narrowed, which signifies the occurrence of random lasing. Furthermore, with increasing the number density of $\mathrm{ZnO}$ and $\mathrm{Ag}$ NPs, the value of the bandwidth is reduced significantly due to enriched multiple scattering. The normalized output characteristics of the binary random lasing system, shown in Fig. 6d, have been significantly affected due to the change in the mixing ratio of the donor and the acceptor. The central wavelength peaks of the emission have shifted positions, indicating that the ET between CT and MB dyes caused switching of the lasing emission by $\sim 30 \mathrm{~nm}$.

The output emission spectra of the binary system were found to be tunable by adjusting the addition of different concentrations of Ag NPs, as shown in Fig. 7a. The normalized emission spectra, as shown in Fig. 7b, clearly show reduction of the lasing threshold from 107 to $25 \mathrm{~W} \mathrm{~cm}^{-2}$, and the threshold value decreased by $\sim 76 \%$ after addition of Ag NPs in the presence of ZnO NPs $\left(n_{\mathrm{ZnO}}=20 \times 10^{12} \mathrm{ml}^{-1}\right)$ in the binary system.

The maximum value of the electric field is located on the surface edges of the Ag NPs. ${ }^{7,8,28}$ Therefore, we believe that this experiment clearly demonstrates that it is possible to achieve low threshold and efficient random lasing by engineering the plasmonic NPs in organic semiconductor thin films. The quality factor ( $Q$-factor) is another important parameter and has been calculated using eqn (4),

$$
Q=\lambda_{0} / \Delta \lambda,
$$

where $\lambda_{0}$ is the central peak wavelength and $\Delta \lambda$ is the mode bandwidth. The value of the $Q$-factor was calculated by fitting the central mode of the emission spectra.


Fig. 7 (a) Scatterer-dependent ASE/lasing spectra of the films; average results in the formation of sharp narrow peaks. (b) The comparison between the effects of injection of Ag NPs on the value of threshold. (c) $Q$-factor as a function of different A/D ratio at the same scatterer concentration. (d) Q-factor as a function of concentration of Ag NPs at $\sim 692.4 \mathrm{~nm}$.
In addition, the $Q$-factor is inversely proportional to the energy loss rate of a mode and is a signature of the lasing threshold for a random system..$^{37}$ Fig. $7 \mathrm{c}$ shows that, as the A/D ratio changed, the value of the $Q$-factor changed from 627 to 1024. Fig. 7d shows the variation in $Q$-factor as a function of Ag $\mathrm{NP}$ concentration, and it was found that $Q=1081$ is the best value for the highest concentration of Ag NPs. This indicates that the presence of both $\mathrm{ZnO}$ and Ag NPs has significantly improved the laser efficiency.

\section{Conclusions}

In conclusion, here we have reported biocompatible CW RL generation, from the natural light-harvesting bio-antenna of butterfly pea flower extract. The positive influence of metalsemiconductor NPs on the bio-pigment leads to enhanced lasing emission at $\sim 660 \mathrm{~nm}$. In addition, RET-based tunable NIR random lasing is demonstrated at $\sim 692 \mathrm{~nm}$ by using the natural dye, i.e. CT extract, and the common, commercially available MB dye. It has been shown that significant reduction in lasing threshold, band narrowing, wavelength tunability in $\mathrm{RL}$ emission of over $30 \mathrm{~nm}$ from 660 to $692 \mathrm{~nm}$ and generation of lasing mode are possible by addition of Ag NPs into the ZnOdoped CT-MB binary system, which is due to the enhancement in the local electric field in triangular-shaped Ag NPs. The biocompatible random laser developed here could provide a new tool for imaging in biological tissues. In addition, we believe that our findings offer a cost-effective approach for optimizing a tunable multi-colour plasmonic RL, avoiding the need to resort to specially engineered, expensive and toxic dyes.

\section{Experimental section}

\section{Materials and synthesis procedure for $\mathrm{ZnO}$ and $\mathrm{Ag}$ nanoparticles}

Zinc nitrate hexahydrate, (Aldrich) potassium hydroxide (Sigma), MB dye (Fluka), silver nitrate and polyvinyl alcohol (PVA) (Sigma) were reagent grade and were used as received without further purification. The synthesis of zinc oxide (ZnO) NPs was carried out by the solvothermal route. First, $4.0 \mathrm{M}$ solution of potassium hydroxide (KOH) was added drop-wise to a $0.5 \mathrm{M}$ solution of zinc nitrate hexahydrate $\left(\mathrm{Zn}\left(\mathrm{NO}_{3}\right)_{2} \cdot 6 \mathrm{H}_{2} \mathrm{O}\right)$ under vigorous stirring to obtain a turbid solution. The solution was then put into an autoclave and maintained at $130{ }^{\circ} \mathrm{C}$ for $8 \mathrm{~h}$. After completion, the autoclave was allowed to cool and the precipitate separated by centrifugation and washing with deionized (DI) water and then dried at room temperature for $24 \mathrm{~h}$. Colloidal triangular silver nanoparticles were synthesized by chemical techniques using a similar procedure to that used previously in our group ${ }^{38}$ (for details see ESI $\dagger$ ).

\section{Extraction of pigments from flowers}

Extraction of pigments from Clitoria ternatea petals was carried out using a very simple method, as described below. In brief, the petals were cut into small pieces and dipped into a beaker with a certain volume of DI water. After $24 \mathrm{~h}$, the blue-coloured 
solution was separated from the mixture using a filter paper (pore diameter $\sim 50 \mu \mathrm{m}$ ) and then was collected in a separate test tube. The collected blue-coloured solution contained flavonoids, anthocyanins, polyphenolic compounds, monoglycosides, diglycosides, etc., as confirmed by FTIR measurement.

\section{Sample characterizations}

The morphology and microscopic structures of $\mathrm{ZnO}$ and $\mathrm{Ag}$ samples were observed using field emission scanning electron microscopy (FESEM, Zeiss) with an acceleration voltage of 5-10 $\mathrm{kV}$, and by transmission electron microscopy (TEM, JEOL JEM, acceleration offset voltage $200 \mathrm{kV}$ ). The sizes of the $\mathrm{ZnO}$ and $\mathrm{Ag}$ NPs were measured from the FESEM and TEM images. The UVvisible absorption spectra were measured in a spectrophotometer (Hitachi U-3010, wavelength range 200-800 $\mathrm{nm}$ ). The photoluminescence (PL) emission properties were determined using a PL spectrofluorometer (PerkinElmer LS-55).

\section{Preparation of polymer film}

First, a clear solution of PVA hydrogel mixture was prepared with CT and MB binary dye mixtures and NPs of ZnO and Ag. Then, PVA solutions were drop-casted onto a glass substrate and left to dry under a controlled atmosphere. The dried flexible film had a thickness of $\sim 68 \mu \mathrm{m}$, measured from the FESEM image (see inset in Fig. 4a).

\section{Random lasing experiment}

Fig. 4a shows a schematic of the experimental set up for measuring RL emission characteristics. The prepared polymer film was pumped by a CW He-Ne laser (power of $\sim 5 \mathrm{~mW}$ ) with a wavelength of $632.8 \mathrm{~nm}$, and with the surface of the film placed at the focus of a lens with focal length $10 \mathrm{~cm}$. The RL emission spectra were captured at an angle of $60^{\circ}$ using an optical spectrometer (Avaspec-2048) through an optical fiber. Finally, all RL emission data were recorded and analyzed by computer.

\section{Conflicts of interest}

There are no conflicts to declare.

\section{Acknowledgements}

The authors are grateful to the Department of Science \& Biotechnology, Government of West Bengal for the partial financial support through project Grant No.: Grant-in-Aids no. 332(Sanc.)/ST/P/S and T/16G-24/2018 dt. 19.03.2019. Partha Kumbhakar is grateful to TEQIP-III, CoE, GOI and MHRD, NIT Durgapur, Government of India for providing a maintenance fellowship.

\section{References}

1 D. S. Wiersma, Nat. Phys., 2008, 4, 359-367.
2 B. Redding, M. A. Choma and H. Cao, Nat. Photonics, 2012, 6, 496.

3 Y. Liu, W. Yang, S. Xiao, N. Zhang, Y. Fan, G. Qu and Q. Song, ACS Nano, 2019, 13, 10653-10661.

4 E. Heydari, I. P. Santos, L. M. L. Marzan and J. Stumpe, Nanoscale Horiz., 2017, 2, 261-266.

5 X. Meng, K. Fujita, S. Murai, T. Matoba and K. Tanaka, Nano Lett., 2011, 11, 1374-1378.

6 Z. Wang, Y. Wang, X. Shi, D. Liu, R. Yu, S. Wei, Z. Lin Wang and Q. Chang, Nanoscale, 2015, 7, 15091-15098.

7 W. Zhang, N. Cue and K. M. Yoo, Opt. Lett., 1995, 20, 10231025.

8 S. Biswas and P. Kumbhakar, Nanoscale, 2017, 9, 1881218818.

9 J. Q. Grim, S. Christodoulou, F. D. Stasio, R. Krahne, R. Cingolani, L. Manna and I. Moreels, Nat. Nanotechnol., 2014, 9, 891-895.

10 V. S. Gummaluri, S. R. Krishnan and C. Vijayan, Opt. Lett., 2018, 43, 5865-5868.

11 M. Athanasiou, R. Smith, B. Liu and T. Wang, Sci. Rep., 2014, 4, 7250 .

12 M. A. Noginov, I. N. Fowlkes, G. Zhu and J. Novak, Phys. Rev. A, 2004, 70, 043811-043816.

13 R. Steiner, H. P. Berlien and G. H. Muller, Applied Laser Medicine, Springer-Verlag, 2003, pp. 101-106.

14 H. Jelinkova, Woodhead Publishing Series in Electronic and Optical Materials Publisher, Woodhead Publishing, 2013.

15 F. Lahoz, I. R. Martín, M. Urgellés, J. M. Alonso, R. Marín, C. J. Saavedra, A. Boto and M. Díaz, Laser Phys. Lett., 2015, 12, 045805-045811.

16 D. Wang, Y. Wang, W. Wang, D. Luo, U. Chitgupi, J. Geng, Y. Zhou, L. Wang, J. F Lovell and J. Xia, Biomed. Opt. Express, 2017, 8, 113.

17 G. Calogero, A. Bartolotta, G. D. Marco, A. D. Carlo and F. Bonaccorso, Chem. Soc. Rev., 2015, 44, 3244-3294.

18 R. Croce and H. Van Amerongen, Nat. Chem. Biol., 2014, 10.

19 J. M. Kong, L. S. Chia, N. K. Goh, T. F. Chi and R. Brouillard, Phytochemistry, 2003, 64, 923-933.

20 T. Fossen, L. Cabrita and O. M. Andersen, Food Chem., 1998, 63, 435-440.

21 A. G. Ardakani and P. Rafieipour, J. Opt. Soc. Am. B, 2018, 35, 1708-1716.

22 R. C. Polson and Z. V. Vardeny, Appl. Phys. Lett., 2004, 85, 1289-1291.

23 L. Ye, B. Liu, F. Li, Y. Feng, Y. Cui and Y. Lu, Laser Phys. Lett., 2016, 13, 105001-105008.

24 L. Cerda, E. Enciso, V. Martin, J. Banuelos, I. L. Arbeloa, A. Costela and I. G. Moreno, Nat. Photonics, 2012, 6, 621-626.

25 J. F. G. Lopez and M. I. C. Lopez, J. Phys. Chem. C, 2014, 118, 9665-9669.

26 C. S. Wang, Y. L. Chen, H. Y. Lin, Y. T. Chen and Y. F. Chen, Appl. Phys. Lett., 2010, 97, 191104-191107.

27 X. Shi, J. Tong, D. Liu and Z. Wang, Appl. Phys. Lett., 2017, 110, 171110.

28 R. Zhang, S. Knitter, S. F. Liew, F. G. Omenetto, B. M. Reinhard, H. Cao and L. Dal Negro, Appl. Phys. Lett., 2016, 108, 011103. 
29 H. Cao, Y. G. Zhao, S. T. Ho, E. W. Seelig, Q. H. Wang and R. P. H. Chang, Phys. Rev. Lett., 1999, 82, 2278.

30 T. Zhai, X. Zhang, Z. Pang, X. Su, H. Liu, S. Feng and L. Wang, Nano Lett., 2011, 11, 4295-4298.

31 Y. Wan, Y. An and L. Deng, Sci. Rep., 2017, 7, 16185-16192. 32 H. Cao, J. Phys. A: Math. Gen., 2005, 38, 10497-10535.

33 J. R. Lakowicz, Principles of Fluorescence Spectroscopy, Springer-Verlag, Berlin, 3rd edn, 2006.

34 X. Wu, W. Fang, A. Yamilov, A. A. Chabanov, A. A. Asatryan, L. C. Botten and H. Cao, Phys. Rev. A: At., Mol., Opt. Phys., 2006, 74, 053812-053823.
35 X. Meng, K. Fujita, S. Murai and K. Tanaka, Phys. Rev. A: At., Mol., Opt. Phys., 2009, 79, 053817-053824.

36 Y. Zhang and S. R. Forrest, Phys. Rev. B: Condens. Matter Mater. Phys., 2011, 84, 241301-241304.

37 P. Wang, X. Zhang, M. Gavryliak, Y. Xiang and J. Xu, Opt. Express, 2015, 23, 24407-24415.

38 K. Mondal, S. Biswas and P. Kumbhakar, Plasmonics, 2019, DOI: $10.1007 / \mathrm{s} 11468-019-01016-6$. 